\title{
Assistência ao recém-nascido prematuro e família no contexto da COVID-19
}

Care for premature newborns and family in the context of the COVID-19

Atención para recién nacidos prematuros y familiares en el contexto de la COVID-19

Andréia Cascaes Cruz ${ }^{1}$ (D) https://orcid.org/0000-0003-2264-0140

Mayrene Dias de Sousa Moreira Alves ${ }^{2}$ (D) https://orcid.org/0000-0002-9397-6517

Bruna Hinnah Borges Martins de Freitas ${ }^{2}$ (D) https://orcid.org/0000-0002-2121-1785

Maria Aparecida Munhoz Gaíva² (D https://orcid.org/0000-0002-8666-9738

\section{Resumo}

Objetivo: Discorrer sobre a assistência ao recém-nascido prematuro e família no contexto da COVID-19.

Métodos: Ensaio teórico-reflexivo fundamentado em evidências atuais e nos princípios do Modelo de Cuidado Centrado no Paciente e Família.

Resultados: A assistência aos neonatos prematuros no contexto da COVID-19 visa a prevenção, o controle, a detecção precoce e o tratamento oportuno dos infectados, além da incorporação de estratégias que possibilitem, sobretudo, a participação efetiva da família na assistência prestada.

Conclusão: Os efeitos da COVID-19 nos prematuros ainda não estão bem esclarecidos. Cabe aos profissionais de saúde prezar por um cuidado seguro, de qualidade e centrado nas necessidades do neonato e de sua família durante a pandemia.

\section{Abstracts}

Objective: To discuss about care for premature newborns and their families in the context of COVID-19.

Methods: Theoretical-reflective essay based on current evidence and the principles of the Patient and Family Centered Care Model.

Results: Nursing care for premature newborns in the context of COVID-19 aims at prevention, control, early detection and timely treatment of those infected, in addition to the incorporation of strategies that enable, above all, the effective participation of the family in the care provided.

Conclusion: The effects of COVID-19 on premature infants are still unclear. It is up to health care professionals to care for safe, quality care and focused on the needs of the newborn and his family during the pandemic.

\section{Resumen}

Objetivo: Discutir la atención de salud para recién nacidos prematuros y sus familias en el contexto de COVID-19. Métodos: Ensayo teórico-reflexivo basado en la evidencia actual y los principios del modelo de atención centrada en el paciente y la familia.

Resultados: La atención de enfermería a los recién nacidos prematuros en el contexto de COVID-19 tiene como objetivo la prevención, el control, la detección temprana y el tratamiento oportuno de los infectados, además de la incorporación de estrategias que permiten, sobre todo, la participación efectiva de la familia en la atención brindada.

Conclusión: Los efectos de COVID-19 en bebés prematuros aún no están claros. Depende de profissionales de la salud una atención segura y de calidad y centrarse en las necesidades del recién nacido y su familia durante la pandemia.

\section{Descritores}

Recém-nascido prematuro; Família; Infecções por coronavírus; COVID-19

\section{Keywords}

Infant, premature; Family; Coronavirus infections; COVID-19

\section{Descriptores}

Recien nacido prematuro; Familia; Infecciones por coronavirus; COVID-19

\section{Como citar:}

Cruz AC, Alves MD, Freitas BH, Gaíva MA. Assistência ao recém-nascido prematuro e família no contexto da COVID-19. Rev Soc Bras Enferm Ped. 2020;20(Especial COVID-19):49-59. 


\section{Introdução}

O novo coronavírus (SARS-COV-2), que surgiu na China no final de 2019, espalhou-se por diversos países se tornando uma emergência de saúde pública internacional. A COVID-19, doença causada por esse vírus, pode se manifestar de diversas formas, desde sintomas respiratórios leves até a síndrome da angústia respiratória aguda grave, com sinais clínicos e radiológicos de pneumonia bilateral. Em 14 de julho de 2020 já haviam sido registradas 12.964 .809 pessoas com COVID-19 e 570.288 óbitos no mundo. ${ }^{(1)} \mathrm{O}$ Brasil, por sua vez, até a mesma data, havia confirmado a doença em 1.926 .824 pessoas, com 74.133 óbitos. ${ }^{(2)}$

A infecção perinatal pelo vírus pode ter efeitos adversos em recém-nascidos ( $\mathrm{RN})$, causando problemas como sofrimento fetal, trabalho de parto prematuro, dificuldade respiratória, trombocitopenia acompanhada de função hepática alterada e até a morte. ${ }^{(3)}$ Estudo identificou que $21,2 \%$ de gestantes com COVID-19 tiveram parto prematuro, no entanto, apenas 6,1\% foram espontâneos. ${ }^{(4)}$ Contudo, estudo de revisão apontou que o nascimento prematuro ocorre em cerca de $47 \%$ das mulheres hospitalizadas com COVID-19 e os achados clínicos nestes RN podem variar desde assintomáticos até manifestações como o desconforto respiratório, que requer suporte ventilatório e demais cuidados intensivos. ${ }^{(5)}$ Portanto, os RN nascidos de mães com COVID-19 confirmado devem ser considerados "pessoas sob investigação".

Deve-se suspeitar da doença em neonatos quando houver presença de pelo menos um dos seguintes sinais ou sintomas clínicos: instabilidade térmica, hipoatividade, dificuldade de alimentação, dificuldade respiratória, radiografia de tórax com alterações; quando houver um familiar/cuidador com COVID-19 ou em caso de contato próximo com pessoas com suspeita ou confirmação da doença. ${ }^{(6)}$

Face a essa conjuntura, o enfermeiro possui papel fundamental na prevenção e controle da infecção, já que o recém-nascido pré-termo (RNPT) é totalmente dependente dos cuidados da equipe de enfermagem, a qual o assiste 24 horas por dia. Além de prevenir a ocorrência da doença, o enfermeiro precisa estar apto para a detecção e o gerenciamento precoces dos casos com uma resposta rápida e eficaz durante a assistência de enfermagem obstétrica e neonatal. ${ }^{(7)}$
Pesquisa de revisão que mapeou as medidas de prevenção e controle da COVID-19 a serem implementadas durante a assistência obstétrica e neonatal, destacou dentre as principais, o uso de máscaras por pessoas suspeitas ou infectadas no contato com neonatos saudáveis; higienização das mãos antes e após cada cuidado e mamada, assim como dos utensílios utilizados para ordenha; uso dos equipamentos de proteção individual pelos profissionais de saúde e; manutenção de quarto privativo para neonatos infectados ou uso de barreiras físicas. Além disso, aponta que o diagnóstico precoce e manejo oportuno dos casos é fundamental para a redução da transmissibilidade do vírus e reabilitação do cliente pediátrico. ${ }^{(7)}$

Incentiva-se a estreita cooperação entre os departamentos obstétrico e pediátrico para oferecer os cuidados necessários aos RNPT com suspeita ou confirmação da infecção por COVID-19, tanto na sala de parto, quanto na Unidade de Terapia Intensiva Neonatal (UTIN). Considerando que o vírus tem um potencial patogênico para causar graves resultados adversos maternos e perinatais, recomenda-se a triagem sistemática de qualquer suspeita de infecção durante a gravidez e o acompanhamento intensivo para mães diagnosticadas e seus filhos. ${ }^{(3)}$

Além do mais, os profissionais de saúde precisam assegurar os cuidados a esses RNPT no ambulatório de seguimento, na Atenção Primária em Saúde (APS) e no domicílio, a fim de acompanhar os eventos adversos da doença a médio e longo prazo, monitorar o crescimento e desenvolvimento e adotar as condutas necessárias para o desenvolvimento pleno desses neonatos. Essa assistência requer uma abordagem humanizada, centrada no RN e na sua família, com intuito de promover a saúde e o bem-estar dos sujeitos do cuidado e restaurar suas condições clínicas.

Neste sentido, apoiado em evidências atuais e no Modelo de Cuidado Centrado no Paciente e Família, este ensaio teórico-reflexivo se propôs a discorrer sobre a assistência ao recém-nascido prematuro e família no contexto da COVID-19. Anseia-se que o mesmo subsidie a efetivação de boas práticas na assistência obstétrica e neonatal.

Para tanto, este ensaio foi organizado em quatro tópicos concernentes, a saber: A COVID-19 em recém-nascidos prematuros; Recomendações de cuidados ao prematuro no nascimento; Recomendações de cuida- 
dos ao prematuro na UTIN; Recomendações de cuidados ao prematuro na Unidade Básica de Saúde/Estratégia Saúde da Família e; Reflexões sobre o cuidado centrado no RNPT e família em tempos de COVID-19.

\section{A CoVID-19 em recém- nascidos prematuros}

Estabelecer discussões sobre os cuidados ao RNPT em meio a pandemia da COVID-19 é essencial, dada a ocorrência de nascimentos prematuros em gestantes infectadas por essa doença. Na maioria dos casos de grávidas contaminadas por coronavírus, o sofrimento fetal intrauterino é encontrado, e essa condição leva a necessidade de interrupção da gestação, muitas vezes prematuramente, para melhor prognóstico materno e neonatal. ${ }^{(3,8)}$ Cabe ressaltar que o sofrimento fetal intrauterino e o parto prematuro nem sempre podem estar diretamente relacionados à infecção por SARS-CoV-2, por isso mais estudos são necessários para confirmar essa correlação. ${ }^{(3)}$

É importante destacar que a infecção por si só não é indicação para interrupção da gestação e nem para indicação do tipo de parto. Sendo assim, as decisões relacionadas a esses fatores devem ser individualizadas junto às famílias. Na maioria dos casos a melhora da condição materna irá estabilizar a condição fetal, permitindo que a gestação chegue ao termo e ao parto vaginal espontâneo. No entanto, se a mulher apresentar condições clínicas graves, que podem levar à morte fetal intrauterina ou perda de mãe e bebê, a gravidez deve ser interrompida e a cesariana realizada. ${ }^{(9)}$

Nos casos de RNPT contaminados por SARS-CoV-2, mesmo que extremamente prematuros, a literatura atual indica que estes não apresentam sintomas clínicos graves, ${ }^{(8)}$ porém os danos potenciais dessa nova doença ainda permanecem desconhecidos. ${ }^{(10)}$ Quanto às manifestações clínicas nos RNPT, estas podem ser inespecíficas, e incluem síndrome do desconforto respiratório agudo, instabilidade térmica e gastrointestinal e disfunção cardiovascular. ${ }^{(10)}$

Cabe ressaltar que os RNPT se encontram em risco particular de infecção perinatal por COVID-19, devido ao ambiente fetal e imaturidade imunológica passiva, além da integridade imatura da pele, podendo ser um fator de risco para transmissão perinatal do vírus. ${ }^{(11)}$ Considerando as particularidades do RNPT, é necessário que haja atenção redobrada, além do conhecimento das medidas de prevenção e controle para proteger e promover a saúde dessa população no contexto da pandemia.

A transmissão da COVID-19 aos RN ocorre principalmente por meio de contato próximo, transmissão por gotículas, infecções adquiridas em hospitais e exposição a fontes de infecção em locais públicos. Até o momento, as evidências não confirmaram a transmissão vertical e infecção intrauterina por SARS-CoV-2 em fetos de mulheres que desenvolveram pneumonia por COVID-19 no final da gravidez. ${ }^{(10)}$ Ademais, ainda não foram encontrados indícios do vírus nas amostras de líquido amniótico, sangue do cordão umbilical, swab neonatal ou amostra de leite materno. ${ }^{(3,8)}$ Entretanto, por se tratar de uma situação nova , as evidências ainda estão sendo produzidas, portanto esses dados podem ser atualizados em breve.

Os pesquisadores que defendem a ausência da transmissão vertical em gestantes contaminadas pelo COVID-19, justificam que durante o pico de infecção não há tempo hábil para a produção materna de anticorpos e a imunidade passiva se desenvolver e, portanto, o feto não entra em contato com a doença. (3) Nesse sentido, reforça-se a recomendação de que o parto só deve ser antecipado em casos de risco-benefício para a mãe ou quando o feto necessitar de alguma intervenção.

Como a forma de transmissão do SARS-CoV-2 ao RNPT parece ser a horizontal, é imprescindível que se aperfeiçoe e otimize os métodos de prevenção da disseminação do vírus nos setores de atendimento ao RNPT, unidades de trabalho de parto e parto, maternidades, enfermarias neonatais e unidades de terapia intensiva, ${ }^{(8)}$ bem como, ofereça atendimento baseados nas melhores evidências. Neste sentido, apresentaremos a seguir algumas recomendações sobre o atendimento ao RNPT no contexto da pandemia por COVID-19, com base nas evidências disponíveis.

\section{Recomendações de cuidados ao prematuro no nascimento}

Os cuidados na sala de parto aos neonatos nascidos de mães assintomáticas e que afirmam ausência de con- 
tato com pessoas com infecção pelo COVID-19 ou de sintomas respiratórios, permanecem os mesmos preconizados nos protocolos de assistência ao nascimento. No Brasil, destacam-se as normas do Programa de Reanimação Neonatal ${ }^{(12,13)}$ da Sociedade Brasileira de Pediatria (SBP).

Os cuidados na sala de parto aos RNPT de mães sintomáticas ou que tiveram contato com uma pessoa com sintomas semelhantes aos da gripe ou confirmado para COVID-19 deve primar pela não exposição ao vírus. Algumas recomendações ainda não são totalmente concludentes, dada a falta de evidências sobre essa nova doença, ao passo que, geralmente, elas são baseadas na opinião de especialistas e em experiências a partir dos casos atendidos. Assim, ainda não há consenso sobre o corte tardio do cordão umbilical, contato pele a pele e amamentação na primeira hora de vida.

O clampeamento tardio do cordão umbilical é recomendado aos RNPT que não necessitam de reanimação após o nascimento, devido aos diversos benefícios já conhecidos. ${ }^{(13)}$ Porém, em neonatos nascidos de mães com suspeita ou confirmada da COVID-19, as recomendações são divergentes. Alguns relatórios baseados em opiniões de especialistas não recomendam esse procedimento com o argumento de se reduzir o risco de transmissão vertical. ${ }^{(3,14)}$

Por sua vez, o Colégio Real de Obstetras e Ginecologistas (RCOG) e a Academia Americana de Pediatria (AAP) aconselham o corte tardio do cordão umbilical. ${ }^{(15,16)}$ Essa indicação está sustentada no fato de que se foi realizado parto vaginal com exposição à secreção materna e sangue, um minuto de perfusão adicional de sangue placentário provavelmente não altera o risco de transmissão vertical. ${ }^{(5)}$ Além disso, ressalta-se que o feto trocou o mesmo suprimento sanguíneo materno durante toda a gravidez, e se a transmissão vertical fosse possível, esta ocorreria provavelmente antes do parto e não nos 60 segundos após o nascimento. ${ }^{(17)}$

Em relação ao corte tardio do cordão umbilical, é preciso considerar os seus diversos benefícios, principalmente aos RNPT, melhor transição circulatória e estabelecimento do volume de glóbulos vermelhos, redução da necessidade de transfusão sanguínea, menor incidência de enterocolite necrotizante e hemorragia intraventricular. ${ }^{(18)}$ Ao passo que, tais benefícios parecem superar o risco improvável de adquirir a COVID-19 por meio do corte tardio do cordão umbilical. ${ }^{(17)}$ No Brasil, a SBP recomenda que em $\mathrm{RN} \leq$ de 34 semanas que respirou ou chorou ao nascer e se encontra ativo, deve-se aguardar 30-60 antes de clampear o cordão umbilical, porém o neonato não deve ser posicionado no abdome ou tórax materno durante esse período, a fim de não haver o contato pele a pele (CPP) com a mãe. ${ }^{(19)}$

Em relação a isso, algumas diretrizes e consensos também discordam sobre a realização do CPP em caso de puérperas sintomáticas ou que teve contato com uma pessoa com sintomas semelhantes aos da gripe ou confirmado para COVID-19. Especialistas chineses não recomendam esse procedimento a fim de evitar a exposição do bebê com a pele da mãe no pós-parto. ${ }^{(14)}$

A $\mathrm{AAP}^{(16)}$ afirma que, se forem tomadas todas as precauções de controle de infecção (incluindo o uso materno da máscara cirúrgica) e a mãe puder, ela poderá segurar brevemente o bebê durante o clampeamento tardio do cordão umbilical. Porém, orienta que os RN sejam lavados após o nascimento para remover vírus potencialmente presentes nas superfícies da pele. Já a OMS preconiza o incentivo e a facilitação do CPP precoce e ininterrupto entre mães e bebês logo após o nascimento, aplicando as medidas necessárias de prevenção e controle da infecção.(1)

A SBP aconselha que não haja CPP e que, após o clampeamento tardio, todos neonatos sejam levados a mesa de reanimação em campos aquecidos, para a realização dos procedimentos de rotina dos serviços. Nos RN > 34 semanas com boa vitalidade ao nascer, a amamentação e o CPP devem ser adiados até o momento em que seja realizado os cuidados de higiene da puérpera, tais como banho de leito, troca de máscara, touca, camisola e lençóis. ${ }^{(19)}$ Os $\mathrm{RN}<34$ semanas, devem ser envoltos em saco plástico transparente e colocado touca dupla, e se não precisar de suporte para a sua transição e estiver respirando de maneira rítmica e regular, com frequência cardíaca > 100 batimentos/minuto, pode-se proceder aos procedimentos de rotina de acordo cada serviço. ${ }^{(19)}$

O banho imediato do RN com boa vitalidade ao nascer após o nascimento, para limpeza dos possíveis vírus presentes nas superfícies da pele naqueles nascidos de mães contaminadas ou com suspeita de COVID-19, ainda é controverso. As evidências relacionadas ao seu papel protetor são incipientes, porém Organizações Internacionais sugerem o banho precoce 
naqueles neonatos nascidos de parto vaginal e expostos ao períneo materno, visto os achados de viremia em fezes detectados em adultos com COVID-19. No Brasil, a recomendação é de que a indicação do banho na primeira hora de vida seja individualizada, considerando as condições de cada serviço de saúde. ${ }^{(19)}$

No tocante ao aleitamento materno na primeira hora de vida, até o presente momento os estudos não evidenciaram a presença do vírus no leite materno de mães confirmadas com COVID-19. No entanto, pesquisadores chineses ainda não descartaram a possibilidade de transmissão vertical da COVID-19 durante a amamentação, por isso não recomendam que os bebês sejam alimentados com leite de mães com diagnóstico confirmado ou com suspeita dessa doença. ${ }^{(14)}$

Contudo, as principais Organizações Internacionais e a Sociedade Brasileira de Pediatria orienta que as mães com COVID-19 podem amamentar seus recém-nascidos, se assim desejarem, porém, devem usar máscaras, e lavar bem as mamas e as mãos antes e depois de tocar nos bebês. ${ }^{(1,15,16)}$ Caso as mães não sintam segurança em amamentar, orienta-se que o leite seja ordenhado e oferecido ao neonato por uma outra pessoa que não apresente sintomas da doença e não tenha tido contato com pessoa doente. Essas recomendações baseiam-se no fato de que os benefícios da amamentação devem pesar contra o risco de transmissão do SARS-CoV-2. ${ }^{(17)}$

Cabe ressaltar que as recomendações com relação ao aleitamento materno na primeira hora de vida são as mesmas que devem ser seguidas durante o período de internação do RN. No caso de separação da mãe e do RN, o uso rotineiro de substitutos do leite materno deve ser evitado. Recomenda-se a ordenha com bomba, transporte e administração do leite da mãe fresco ao recém-nascido, sem a necessidade de pasteurizá-lo. ${ }^{(20)}$ O leite pasteurizado de doador é um recurso vital para bebês, inclusive os prematuros, cujas mães não podem fornecer o seu próprio leite materno. ${ }^{(21)}$

A equipe de saúde no atendimento ao RN no nascimento deve utilizar os Equipamentos de Proteção Individual (EPI) para transmissão por aerossóis, gotículas e contato (máscaras N95 ou PFF2, óculos e protetores faciais, aventais impermeáveis de mangas longas, luvas de procedimentos e gorros) pela possibilidade de aerossóis com vírus materno e necessidade potencial de in- tubação traqueal, aspiração de vias aéreas e ventilação com pressão positiva que também pode gerar aerossóis para o RN. ${ }^{(19)}$ Além disso, reforça-se a necessidade de higienização frequente das mãos com água e sabonete líquido e preparação alcoólica (70\%). ${ }^{(19)}$

Outro aspecto importante a se destacar no atendimento ao RN na sala de parto, considerando os dados atuais que indicam a relação entre parto prematuro em mulheres com COVID-19 confirmado, é a necessidade de profissional habilitado para o atendimento do bebê, principalmente em reanimação neonatal, a fim de melhorar a taxa de sobrevida e o prognóstico. Faz-se necessário equipe adequada, porém mínima, para evitar a chance de exposição de profissionais de saúde ao vírus. ${ }^{(19)}$

Todas as etapas da reanimação de RNPT nascido de mãe suspeitas ou contaminadas pelo COVID-19 devem seguir as mesmas diretrizes dos Programas de Reanimação empreendida pelo serviço. No Brasil, utiliza-se as diretrizes da SBP de 2016 embasadas no Manual de Reanimação Neonatal da AAP. (19)

Idealmente, a área para a realização da reanimação de neonatos nascidos de mães suspeitas ou confirmada de COVID-19 deve ser em um local separado e com pressão negativa no ar, isolada para garantir a mínima exposição possível do RN e equipe. Caso não disponha de sala com isolamento de pressão pode-se instalar dois exaustores. Se não for possível sala isolada, o berço aquecido para reanimação deve ser separado da área de parto a uma distância de pelo menos 2 metros entre a mãe e a mesa de reanimação. Uma cortina estéril impermeável pode ser usada entre as duas áreas para minimizar exposição. ${ }^{(17)}$

No Brasil, a disponibilidade de salas com pressão negativas é reduzida, nesse sentido a sugestão é de que, sempre que possível, seja preparado previamente o fluxo de entrada de pessoas, equipamentos, limpeza e descontaminação. Se plausível utilizar uma sala separada daquela onde aconteceu o parto, ou manter a distância entre a mãe e a mesa de reanimação neonatal. ${ }^{(19)}$

Os materiais necessários para o atendimento de neonatos nascidos de mães com suspeita ou confirmação de infecção pela COVID-19 devem ser checados e testados antes do nascimento, e são os mesmos indicados pelo Programa de Reanimação Neonatal. Em caso de necessidade de material extra, sugere-se que este seja trazido por um profissional que esteja fora da sala, 
para evitar que a equipe que está no atendimento ao RN saia da sala de parto. ${ }^{(19)}$

O RNPT de mãe com suspeita ou confirmação de COVID-19 deve ser transportado em uma incubadora de transporte própria e de acordo com o protocolo e fluxo de cada instituição. ${ }^{(19)}$ A limpeza adequada da incubadora após a sua utilização é essencial e deve ser realizada segundo as normas da Comissões de Controle de Infecção institucional. ${ }^{(19)}$

Considerando que todos os RN suspeitos ou confirmados para a COVID-19 necessitam ser internados na UTIN, ${ }^{(22)}$ é fundamental que os profissionais de saúde que atuam nessas unidades conheçam as recomendações de cuidados sustentadas em evidências científicas.

\section{Recomendações de cuidados ao prematuro na UTIN}

O Centro de Controle e Prevenção de Doenças recomenda a separação de RNPT de mães com suspeita ou confirmação de COVID-19. Um teste de SARS-CoV-2 deve ser realizado no neonato após o nascimento, ${ }^{(10)}$ os testes indicados são esfregaços nasofaríngeos e na garganta 24 horas após o nascimento e repetidos 48 horas após o nascimento. ${ }^{(23)}$ De acordo com o Ministério da Saúde, a triagem laboratorial para investigação de SARS-CoV-2 em RN assintomático cuja mãe tenha diagnóstico suspeito ou confirmado de COVID-19 não é necessária, mas poderá ser realizada se houver disponibilidade e logística para tal no serviço de saúde. ${ }^{(22)}$

Recomenda-se que todos os RNPT suspeitos ou confirmados permaneçam internados na UTIN e isolados por 14 dias. O isolamento dos RN prováveis ou confirmados em laboratório com SARS-CoV-2 deve ser realizado em um único quarto (se possível), equipado com um sistema de ciclo de ar isolado e com pressão negativa. ${ }^{(22)}$

Face a necessidade de isolamento dos recém-nascidos prematuros suspeitos ou confirmados com COVID-19 na UTIN, medidas de precaução padrão são recomendadas, dentre as quais destacamos no Quadro 1.

Medidas padrão e adicionais de controle de infecção devem ser implementadas imediatamente, ${ }^{(24)}$ como políticas de restrição de visitantes. ${ }^{(10)}$ Mães ou pais sintomáticos, ou que tiveram contato com indiví-
Quadro 1. Medidas de precaução padrão para recém-nascidos prematuros suspeitos ou confirmados com COVID-19 na UTIN

1. Manter o RNPT em incubadora, utilizando precaução de contato, gotículas e/ou aerossóis de acordo com o tipo de procedimento a ser realizado.

2. Executar limpeza frequente da incubadora fixa e de transporte após cada uso.

3. Realizar procedimentos de intubação, administração de surfactante, passagem de sonda orogástrica e aspiração orotraqueal com cuidados intensificados para proteção, acrescentando o uso de máscara N95/PFF2 e óculos de proteção.

4. Proceder aspiração de cânula orotraqueal, preferencialmente, com sistema fechado.

5. Trocar roupas e luvas após os procedimentos com os RNPT.

6. Efetuar desinfecção terminal do quarto do paciente preferencialmente usando atomização por peróxido de hidrogênio ou um spray de preparação contendo cloro.

7. Evitar aglomeração de profissionais durante a passagem de plantão e visitas à beira do leito.

Fonte: Sociedade de Pediatria de São Paulo. Recomendações para Cuidados e Assistência ao Recém-Nascido com suspeita ou diagnóstico de COVID-19 - $2^{\text {a }}$ versão atualizada em 06/04/2020. Disponivel em: https://www.spsp.org.br/PDF/COVID\%20 Recomenda\%C3\%A7\%C3\%B5es\%20DC\%20Neo\%20SPSP-6abril2020.pdf ${ }^{\text {(2) }}$

duos sintomáticos ou positivos para COVID-19, não devem visitar os neonatos hospitalizados até que se tornem assintomáticos e o período de transmissibilidade da COVID-19 tenha passado (aproximadamente 14 dias). ${ }^{(19)}$

A triagem diária de sintomas respiratórios é recomendada para pais e mães que visitam os RN. Nos casos em que o pai ou a mãe não podem permanecer na UTIN, a recomendação é de que a família indique para ficar com o recém-nascido uma pessoa assintomática sem contato doméstico com um indivíduo com sintomas ou positivo para COVID-19. (22)

Diante da ausência de contato do RN com familiares para diminuir o risco de transmissão do SARS-CoV-2, é preciso considerar que estas reduzem as oportunidades dos $\mathrm{RN}$ serem tocados e realizarem o contato pele a pele, cujos benefícios já estão fortemente evidenciados. ${ }^{(21)} \mathrm{A}$ decisão de separar ou não a mãe e o recém-nascido deve ser individualizada, levando em consideração o seu consentimento informado, a logística do hospital e a situação epidemiológica local da pandemia da COVID-19.(20)

Na UTIN, os cuidados ao RNPT sintomático devem estar direcionados ao monitoramento contínuo e estrito (frequência cardíaca, frequência respiratória, saturação de oxigênio, temperatura, pressão arterial, glicemia). Exames de sangue e radiografia de tórax serão necessários, para tanto, a equipe de deve considerar a manipulação mínima do RN, gerenciando o agrupamento da realização de exames clínicos ou complementares e os cuidados. O profissional de saú- 
de deve ainda se atentar para alterações gastrointestinais, passíveis de ocorrer nos casos sintomáticos. ${ }^{(10)}$

Em alguns casos pode ser necessário o fornecimento de suporte respiratório por cânula nasal de alto fluxo, ventilação não invasiva ou ventilação mecânica. ${ }^{(10)}$ Diante disso, os cuidados de saúde devem ser adequados à terapêutica ventilatória instituída, considerando, os aspectos específicos de cada uma, como controle da saturação de oxigênio, conferência dos parâmetros ventilatórios e a avaliação da dor por meio de escalas apropriadas à idade gestacional do RN.

A equipe de saúde deve estar preparada para realizar os cuidados relacionados a terapia de reposição de surfactante, óxido nítrico inalatório e ventilação oscilatória de alta frequência, uma vez que podem ser utilizadas para recém-nascidos com síndrome do desconforto respiratório grave, apesar de não existirem evidências sobre sua eficácia. Ainda, é preciso que os profissionais considerem na gestão dos cuidados, a possibilidade de neonatos gravemente enfermos necessitarem da substituição renal contínua ou da oxigenação por membrana extracorpórea. ${ }^{(10)}$

Quanto aos cuidados relacionados à terapia medicamentosa, a equipe deve estar atenta a prescrição de antibióticos, pois o uso empírico desses fármacos deve ser evitado, devendo ser administrados apenas ao RN com infecção bacteriana confirmada. ${ }^{(10)}$ Uma discussão interprofissional acerca dessa terapêutica pode ser necessária nas visitas clínicas. Não existem evidências sobre a eficácia de medicamentos antivirais para crianças com COVID-19 até hoje. ${ }^{(10)}$

Por ocasião da alta de neonatos nascidos de mães positivas para COVID-19 que apresentarem testagem negativa ou forem assintomáticos, a equipe da UTIN deve informar a família que o cuidado desse bebê precisa ser realizado por uma pessoa não infectada e assintomática. Se o RN que receber alta hospitalar estiver assintomático, mas testou positivo para o vírus, a família deverá providenciar o seu isolamento no domicílio por 14 dias. Reforçar que, se possível, indivíduos não infectados com idade superior a 60 anos ou com comorbidades não devem cuidar desse $\mathrm{RN}$ em casa. ${ }^{(23,24)}$

Os RNPT após a alta hospitalar necessitam ter assegurado o monitoramento de seu crescimento e desenvolvimento e os cuidados para a prevenção e o controle da COVID-19, que estão descritos a seguir.

\section{Recomendações de cuidados ao prematuro nas Unidades Básicas de Saúde/Estratégia Saúde da Família}

O acompanhamento do RN prematuro é parte essencial dos esforços dos profissionais de saúde para garantir os melhores resultados possíveis para as crianças assistidas em UTIN. Além disso, o acompanhamento tem por finalidade a orientação e apoio às famílias para a realização do cuidado aos lactentes no domicílio.

No contexto da pandemia da COVID-19, o Ministério da Saúde ${ }^{(25)}$ sugeriu o adiamento temporário das consultas eletivas para o prematuro assintomático nos ambulatórios de "follow up" no ambiente hospitalar, mas recomendou a manutenção das consultas de seguimento eletivas aos RN na atenção básica, justificada pela oportunidade para a imunização de rotina, vigilância do crescimento e desenvolvimento e orientações à família.

Assim, os RNPT após a alta hospitalar de unidades neonatais e sua família deverão receber o cuidado das equipes da Unidade Básica de Saúde (UBS) ou Estratégia Saúde da Família (ESF), cujos profissionais deverão desenvolver, de forma compartilhada, o cuidado nas consultas, visitas domiciliares, observação e toda orientação necessária nessa situação de pandemia.

O acompanhamento do RNPT, após a alta hospitalar da unidade canguru, tem como objetivo observar a relação pais-bebê, esclarecer dúvidas, estimular a posição canguru e o aleitamento materno. Ressalta-se que, apesar da criança estar clinicamente estável, ela ainda necessita da estabilidade térmica e de ganho de peso, dentre outros cuidados. Ainda, nas primeiras semanas após a saída do hospital, algumas famílias podem precisar de maior apoio, e para as quais a equipe da ESF deve ter uma atenção especial. ${ }^{(26)}$

Para àquelas crianças que os familiares necessitam de maior suporte para os cuidados ou a mãe apresente dificuldades no manejo da amamentação, além da manutenção do cronograma de consultas nas unidades, a equipe de saúde deve lançar mão da visita domiciliária, em especial na primeira semana após a alta hospitalar. 
Considera-se que, o profissional que atua na atenção básica em saúde, em especial na ESF, tem amplo conhecimento do território e das famílias vulneráveis, além do vínculo com os usuários, em especial com as famílias das crianças prematuras. Por isso, ele tem papel importante na orientação das medidas preventivas e de controle da COVID-19 às famílias dessas crianças. Além do mais, pode estabelecer o acompanhamento aos casos suspeitos e leve, garantindo um atendimento seguro e de qualidade neste nível de atenção.

Ademais, as UBS/ESF por serem a porta de entrada do Sistema Único de Saúde (SUS), têm papel de destaque nessa situação de pandemia, com grande potencial de identificação precoce e manejo oportuno dos casos da COVID-19, e encaminhamento dos casos graves para os serviços especializados.

O quadro 2 traz as recomendações para os cuidados no atendimento das crianças prematuras e famílias nas UBS/ESF, nesse contexto da COVID-19.

O seguimento do RNPT é uma extensão do cuidado perinatal, e definido como prioritário, portanto, neste momento de pandemia ele deve ser mantido, desde que sejam realizados ajustes no fluxo e na forma de atendimento. Assim, será possível oferecer uma assistência adequada a todas essas crianças, evitando que adoeçam e necessitem de hospitalização por conta da pandemia.

\section{Reflexões sobre o cuidado centrado no RNPT e família em tempos de COVID-19}

Neste tópico nos propomos a refletir sobre a prestação de um cuidado de saúde que garanta a segurança da equipe, do neonato e da família em termos de contaminação pelo COVID-19, e ao mesmo tempo enfocamos o cuidado desenvolvimental e humanizado, centrado no neonato e em sua família.

Na sala de parto, diante da atual situação de pandemia de COVID-19, e das medidas recomendadas pelas principais Organizações Internacionais, faz-se necessário que haja tomada de decisão compartilhada dos profissionais de saúde com os pais antes do parto, esclarecendo a incipiência das evidências e a explanação de riscos potenciais e benefícios dos procedimentos que serão reali-
Quadro 2. Cuidados ao RNPT nas UBS/ESF

1. Proceder higiene rigorosa das mãos antes e após o manuseio do RN.

2. Uso de luvas de procedimentos e máscara cirúrgica durante 0 atendimento ao RN.

3. Manter estratégias de distanciamento social, cobrir a boca ao tossir ou espirrar com lenços descartáveis; intensificar os esforços de limpeza e desinfecção dos objetos e equipamentos de cuidado utilizados no atendimento ao RN.

4. Limpar e desinfetar objetos e superfícies tocados com frequência.

5. Os equipamentos/materiais como estetoscópio e termômetro devem ser de uso individuais.

6. Os resíduos produzidos durante 0 atendimento ao RN devem ser coletados em um saco de resíduos infecciosos de dupla camada e tratados com cloro por pelo menos 10 minutos e depois descartado da mesma maneira que os resíduos infecciosos.

7. Deve-se adotar rigoroso protocolo de desinfecção de ambientes após atendimento dos RN.

8. Programar os fluxos de atendimento dos neonatos para evitar aglomerações e orientar a família a comparecer na unidade apenas com um acompanhante.

9. Orientar os pais/familiares sobre a importância de distanciamento social, evitando circulação desnecessária em lugares públicos com o bebê prematuro, visitas sociais ao RN, inclusive dos avós, nem mesmo com uso de máscaras; higiene frequente das mãos; cobrir o nariz e boca quando for tossir ou espirrar; e uso de máscara facial em ambientes externos.

10. Esclarecer os pais/familiares da necessidade de manter os cuidados com 0 ambiente, sempre que possível, abrindo janelas e portas para a circulação do ar.

11. Estimular a amamentação, mesmo que a mãe seja caso suspeito ou confirmado de COVID-19, pois não há evidências até o momento de que 0 vírus SARS-CoV2 seja transmitido pelo leite materno.

12. Conscientizar os pais/familiares, que caso algum membro da família ou contatos domiciliares apresente sintomas da COVID-19 ou mesmo manifestações gripais, deve-se evitar contato com o recém-nascido prematuro e com todas as pessoas que moram na mesma casa e/ou que são responsáveis diretas por seu cuidado, por pelo menos 14 dias, período de transmissibilidade da SARS-CoV-2.

13. Orientar os pais/familiares a procurar imediatamente atendimento hospitalar se o RN apresentar algum agravo/intercorrência como: inapetência para amamentação ou ingestão de líquidos, falta de ar ou dificuldade para respirar; ronco, retração sub/intercostal severa; cianose central e batimento da asa de nariz, dentre outros.

14. Ao acolher um RNPT com suspeita de infecção por COVID-19, deve-se fazer a investigação a partir do histórico familiar e clínico, e em caso de exposição a pessoas infectadas, independentemente de seus sintomas, encaminhá-lo para teste.

15. Se possível, manter contato telefônico com a familiares/mãe/cuidador para sanar dúvidas e para propiciar vínculo e coresponsabilização nesse momento de pandemia.

16. Apoiar e sanar as dúvidas da família acerca dos cuidados preventivos para a COVID-19 no domicílio e para com o bebê prematuro.

17. Criar espaço para que a mãe ou familiares relate queixas e/ou intercorrências desde a alta hospitalar, valorizando todas demandas trazidas por ela.

18. Priorizar as visitas domiciliárias, em especial nos primeiros dias após a alta dos RNPT, mantendo todos os cuidados para segurança dos profissionais e da família (EPI). Nessas visitas, utilizar balanças domiciliares para acompanhar 0 ganho de peso dos neonatos, observar as condições da amamentação, a ambiência em casa e orientar a higienização com produtos de forma segura.

Fonte: Adaptado de Brasil. Ministério da Saúde. Secretaria de Atenção Primária à Saúde. Nota Técnica nº 10/2020-COCAM/CGCIVI/DAPES/SAPS/MS. Atenção à Saúde do Recém-nascido no contexto da Infecção pelo novo Coronavírus (SARS-CoV-2), 09 Abr. 2020. ${ }^{(27)}$

zados com o RNPT. Portanto, é importante compreender a visão dos pais/família, considerando a autonomia dos intervenientes frente às necessidades do RNPT e família. 
Também é essencial aos profissionais que assistem o RNPT nascido de mãe suspeita ou confirmada com a COVID-19 na sala de parto, considerem a importância dos benefícios de alguns procedimentos para o bebê e família, principalmente nas primeiras horas de vida, e que os realizem assim que possível, seguindo todas as medidas necessárias para prevenção e controle da infecção. Dentre os referidos procedimentos benéficos estão o aleitamento materno na primeira hora de vida e o CPP mãe-bebê, tendo como consequências promoção de maior vínculo entre essa díade, auxílio na regulação térmica e estabilização dos sinais vitais do RN. Nesse sentido, mesmo diante da pandemia por COVID-19, torna-se fundamental a implementação de estratégias que garantam a realização desses procedimentos, a fim de promover o melhor cuidado ao neonato e sua família.

No contexto das UTIN estamos vivenciando situações nas quais a mãe, o pai ou ambos precisam ser afastados de seus bebês por estarem sintomáticos, conviverem com alguém testado positivo para o vírus ou ainda serem confirmados com COVID-19. Para tanto, precisamos implementar estratégias que propiciem um cuidado exitoso para o RN, família e equipe de saúde. Seguir as recomendações de redução de circulação de pessoas, suspensão das visitas dos avós e dos irmãos e limitação da presença dos pais pode configurar-se como uma ação simples, mas ao mesmo tempo, um retrocesso, diante da vivência em um cenário recente de portas "abertas" para as famílias nas UTIs neonatais brasileiras.

O grande desafio na assistência na UTIN neste momento está em como manter as portas abertas para as necessidades das famílias e dos neonatos, ainda que perante inúmeras barreiras. Tornou-se imperativo pensar em maneiras de implementar o Cuidado Centrado no Paciente e Família (CCPF) - dignidade e respeito, compartilhamento de informações, participação e colaboração- considerando a importância dos estímulos sensoriais e do vínculo afetivo para o desenvolvimento do $\mathrm{RN}$, face à um contexto tão atípico e adverso causado pela pandemia.

Diante da ausência da mãe e do pai do RN, é recomendado considerar a presença de outro membro familiar, de modo que se garanta que o bebê não fique sozinho, sem a presença de uma pessoa que propicie o toque, a fala e o contato com olhar humano contínuo, e que ao mesmo tempo mantenha presente a cultura familiar. A interação e o apoio da equipe ao membro da família que permanecerá e prestará os cuidados ao RN devem ser garantidos. ${ }^{(28)}$

Mantém-se a recomendação de que o menor número possível de profissionais toquem e cuidem do RN. Ademais, orienta-se a manutenção dos mesmos profissionais cuidando dos mesmos neonatos, visto que oferece referência para que o bebê reconheça a rotina de cuidados, a voz e o toque de cada profissional, propiciando segurança e confiança diante de tantas mudanças para o mesmo. ${ }^{(28)}$

Nos casos em que os pais estejam impossibilitados de estarem presentes ou com permanência limitada na UTIN, atenção especial deve ser dada ao princípio do compartilhamento de informações. Evidências mostram que uma das maiores necessidades das famílias que vivenciam situações de doença e hospitalização de suas crianças é receber informações dos profissionais de saúde. ${ }^{(29)}$

Para tanto, uma das estratégias que pode ser utilizada diante da ausência parental é a realização de encontros virtuais com as famílias durante as visitas clínicas multidisciplinares. Tablets e/ou smartphones podem ser utilizados e os encontros podem ser feitos por meio de aplicativos ou plataformas como o WhatsApp ${ }^{\circ}$, Google Meet ${ }^{\bullet}$, Skype ${ }^{\oplus}$, dentre outros. Tais recursos já vêm sendo amplamente utilizados para promover a participação das famílias que não podem estar presentes durante as visitas clínicas com a equipe de saúde, sobretudo, em UTIs Pediátricas.

Considerando ainda a necessidade de separação ou limitação da presença dos pais com o bebê na UTIN, é preciso que os profissionais reflitam sobre maneiras de realizar tomadas de decisão compartilhadas com os pais, descritas na definição do princípio participação do CCPF. O planejamento da assistência ao RN pode ser realizado de forma compartilhada com as famílias por meio de encontros virtuais previamente agendados. Esta seria uma forma de aproximar os pais da equipe e do cuidado, mas, sobretudo, minimizar o impacto negativo do distanciamento na construção da parentalidade e do vínculo com o RN.

A partir da compreensão de que não apenas o neonato, mas todos os membros da família são sujeitos de cuidado, de acordo com a filosofia do CCPF, o impacto e o sofrimento emocional no sistema familiar 
são outros elementos cuja avaliação e intervenção devem ser integradas aos cuidados da equipe de saúde. Deste modo, o uso de um guia que norteie o atendimento da família, ainda que de modo virtual, pode ajudá-lo a realizar o cuidado na prática clínica. A avaliação das necessidades familiares pode ainda demandar o encaminhamento de membros da família para acompanhamento com outros profissionais da saúde, como psicólogos ou psiquiatras. ${ }^{(29)}$

Diante da constatação de que, vivenciando enormes desigualdades quanto ao tempo que os pais podem passar com seus bebês em unidades neonatais durante a pandemia do COVID-19, e que separação de bebês e pais é prejudicial, foi lançada uma campanha pela Aliança Global para o Cuidados do Recém-Nascido (Global Alliance for Newborn Care - GLANCE) "Separação Zero. Juntos por cuidado melhor! Mantenha bebês prematuros e doentes próximos aos pais". Tal campanha já recebeu apoio de diversas organizações, profissionais de saúde e sociedades de diversos países, inclusive da Organização Mundial de Saúde. ${ }^{(30)}$

A campanha está fundamentada nos prejuízos decorrentes da separação dos bebês de seus pais. São destacados os riscos de danos desencadeados pelas políticas de acesso restrito e ausência das famílias nos cuidados neonatais, a curto e a longo prazo. Para evitar tais impactos negativos, é vital que os pais estejam envolvidos na assistência, pois são um componente essencial e insubstituível no cuidado do seu bebê.

Recomenda-se que mesmo em tempos de pandemia por COVID-19, os pais possam ficar por tempo irrestrito e ilimitado com o filho na UTIN. Os benefícios de tal prática estão bem documentados e têm efeitos ao longo da vida do bebê e para os pais, por exemplo as taxas de amamentação são mais altas, melhoram os resultados no desenvolvimento neurológico das crianças e diminuem o tempo de internação hospitalar. Todos esses resultados positivos descritos podem ser alcançados ao permitir o acesso irrestrito dos pais ao bebê na unidade neonatal, juntamente com a participação nos cuidados, educação e apoio psicossocial.

Quanto aos cuidados aos bebês prematuros nas UBS/ESF, além do atendimento presencial com todas as precauções de segurança, os profissionais podem utilizar a teleconsulta ou teleatendimento a fim de sanar dúvidas sobre a COVID-19, bem como, sobre as questões atreladas ao crescimento e desenvolvimento, cuidados domi- ciliares ao neonato, alterações apresentadas pelo neonato e quaisquer outras demandas trazidas pelas famílias.

\section{Considerações finais}

A partir das evidências apresentadas, considera-se que os efeitos da COVID-19 aos RNPT ainda não se encontram bem esclarecidos. Devido a maior vulnerabilidade dos prematuros à infecção, compete à equipe de saúde a implementação de medidas de controle e prevenção da doença nos diferentes ambientes de assistência à saúde. As reflexões trazidas à luz do Modelo de Cuidado Centrado no Paciente e Família destacam o papel da equipe de saúde como agentes de um cuidado pautado no pressuposto de que a família também é sujeito do cuidado, e que a parceria com ela estabelecida resulta em melhores práticas e resultados de saúde. Os cuidados de saúde prestados ao RNPT e família nos diferentes cenários assistenciais visam a prevenção, detecção precoce, tratamento oportuno dos infectados por SARS-COV-2, controle da doença e gerenciamento do cuidado para avaliação e atendimento das necessidades do RNPT e sua família. Salienta-se que tais práticas devem garantir a segurança dos intervenientes e, concomitantemente, considerar a incorporação de novas estratégias que propiciem a implementação do cuidado desenvolvimental, humanizado e centrado no neonato e em sua família, mesmo diante da pandemia por COVID-19.

\section{Referências}

1. World Health Organization (WHO). Coronavirus disease (COVID-19) pandemic. Genève: WH0; 2020 [cited 2020 Jul 15]. Genève:WH0; 2020 [updated 2020 Jul 20; cited 2020]. Available from: https://www.who.int/emergencies/diseases/novel-coronavirus-2019

2. Brasil. Ministério da Saúde. Painel de casos de doença pelo coronavírus 2019 (COVID-19) no Brasil pelo Ministério da Saúde. Brasília (DF): Ministério da Saúde; 2020.

3. Zhu H, Wang L, Fang C, Peng S, Zhang L, Chang G, et al. Clinical analysis of 10 neonates born to mothers with 2019-nCoV pneumonia. Transl Pediatr. 2020; 9(1):51-60.

4. Yan J, Guo J, Fan C, Juan J, Yu X, Li J, et al. Coronavirus disease 2019 in pregnant women: a report based on 116 cases. Am J 0bstet Gynecol. 2020;223:111.e1-1.

5. Mullins E, Evans D, Viner RM, O'Brien P, Morris E. Coronavirus in Pregnancy and Delivery: Rapid Review. Ultrasound Obstet Gynecol. 2020;55(5):586-92.

6. Hong H, Wang Y, Chung HT, Chen CJ. Clinical characteristics of novel coronavirus disease 2019 (COVID-19) in newborns, infants and children. Pediatr Neonatol. 2020;61(2):131-2.

7. Freitas BH, Alves MD, Gaíva MAM. Medidas de prevenção e controle de infecção neonatal por COVID-19: revisão de escopo. Rev Bras Enferm. 2020; 73(Suppl 2):e20200467.

8. Piersigilli F, Carkeek K, Hocq C, van Grambezen B, Hubinont C, Chatzis 0, et al. COVID-19 in a 26-week preterm neonate. Lancet Child Adolesc Health. 2020;4(6):476-8. 
9. Chen D, Yang H, Cao Y, Cheng W, Duan T, Fan C, et al. Expert consensus for managing pregnant women and neonates born to mothers with suspected or confirmed novel coronavirus (COVID-19) infection. Int J Gynaecol Obstet. 2020;149:130-6.

10. De Rose DU, Piersigilli F, Ronchetti MP, Santisi A, Bersani I, Dotta A, et al. Novel Coronavirus disease (COVID-19) in newborns and infants: what we know so far. Ital J Pediatr. 2020;46(1):56.

11. Blauvelt CA, Chiu C, Donovan AL, Prahl M, Shimotake TK, George RB, et al. Acute respiratory distress syndrome in a preterm pregnant patient with coronavirus disease 2019 (COVID-19). Obstet Gynecol. 2020;136(1):46-51.

12. Sociedade Brasileira de Pediatria (SBP). Reanimação do recém-nascido $\geq 34$ semanas em sala de parto: Diretrizes 2016 da Sociedade Brasileira de Pediatria. SBP; 2016 [citado 13 Jun 2020]. Disponível em: https://www.sbp.com.br/fileadmin/user_upload/ DiretrizesSBPReanimacaoRNMaior34semanas26jan2016.pdf

13. Sociedade Brasileira de Pediatria (SBP). Reanimação do prematuro $<34$ semanas em sala de parto: Diretrizes 2016 da Sociedade Brasileira de Pediatria. SBP; 2016 [citado 13 Jun 2020]. Disponivel em: https://www.sbp.com.br/fileadmin/user_upload/ DiretrizesSBPReanimacaoPrematuroMenor34semanas26jan2016.pdf

14. Wang L, Shi Y, Xiao T, Fu J, Feng X, Mu D, et al. Chinese expert consensus on the perinatal and neonatal management for the prevention and control of the 2019 novel coronavirus infection (First edition). Ann Transl Med. 2020;8(3):47.

15. Royal College of Obstetricians and Gynaecologists (RCOG). Coronavirus (COVID-19) Infection in Pregnancy Information for healthcare professionals. Version 10.1. London (UK): Royal College of Obstetricians and Gynaecologists; 2020.

16. American Academy of Pediatrics (AAP). FAQQS: Management of infants bor to mothers with suspected or confirmed COVID-19. Itasca: AAP; 2020 [cited 2020 Jul 18]. Available from: https://services.aap.org/en/pages/2019-novel-coronavirus-COVID-19-infections/ clinical-guidance/faqs-management-of-infants-born-to-COVID-19-mothers/

17. Chandrasekharan P, Vento M, Trevisanuto D, Partridge E, Underwood MA, Wiedeman J, et al. Neonatal resuscitation and postresuscitation care of infants born to mothers with suspected or confirmed SARS-CoV-2 infection. Am J Perinatol. 2020;37(8):813-24.

18. American College of Obstetricians and Gynecologists (ACOG). Delayed Umbilical Cord Clamping After Birth. Committee Opinion No. 684. Washington, DC: American College of Obstetricians and Gynecologists; 2017. Washington, DC: ACOG; 2020 [cited 2020 Jul 18]. Available from: http://www.acog.org/ Resources-And-Publications/Committee-Opinions/ Committee-onObstetric-Practice/Delayed-Umbilical-Cord-Clamping-After-Birth.

19. Sociedade Brasileira de Pediatria (SBP). Nota de alerta: Recomendações para Assistência ao Recém-Nascido na sala de parto de mãe com COVID-19 suspeita ou confirmadaAtualização 2. São Paulo: SBP; 2020.
20. Davanzo R, Moro G, Sandri F, Agosti M, Moretti C, Mosca F, et al. Breastfeeding and coronavirus disease-2019: ad interim indications of the Italian Society of Neonatology endorsed by the Union of European Neonatal \& Perinatal Societies. Matern Child Nutr. 2020;16(3):e13010.

21. Furlow B. US NICUs and donor milk banks brace for COVID-19. Lancet Child Adolesc Health. 2020;4(5):355.

22. Sociedade de Pediatria de São Paulo (SPSP). Recomendações para Cuidados e Assistência ao Recém-Nascido com suspeita ou diagnóstico de COVID-19 - $2^{\text {a }}$ versão - atualizada em 06/04/2020. São Paulo: SPSP; 2020 [citado 2020 Set 25]. Disponível em: https://www. spsp.org.br/PDF/COVID\%20Recomenda\%C3\%A7\%C3\%B5es\%20DC\%20Ne0\%20SPSP6abril2020.pdf

23. Mimouni F, Lakshminrusimha S, Pearlman AS, Raju T, Gallagher PG, Mendlovic J. Perinatal aspects on the COVID-19 pandemic: a practical resource for perinatal-neonatal specialists. J Perinatol. 2020;40(5): 820-6.

24. Wang J, Qi H, Bao L, Li F, Shi F. A contingency plan for the management of the 2019 novel coronavirus outbreak in neonatal intensive care units. Lancet Child Adolesc Health. 2020;4(4):258-9.

25. Brasil. Ministério da Saúde. Secretaria de Atenção Primária à Saúde. Departamento de Ações Programáticas Estratégicas. Nota Técnica $n^{0}$ 6/2020-COCAM/CGCIVI/DAPES/SAPS/ MS. Atenção à saúde do recém-nascido no contexto da infecção pelo novo coronavírus. Brasília (DF): Ministério da Saúde; 2020.

26. Brasil. Ministério da Saúde. Secretaria de Atenção à Saúde. Departamento de Ações Programáticas Estratégicas. Método Canguru: manual da terceira etapa do Método Canguru na Atenção Básica/Ministério da Saúde, Secretaria de Atenção à Saúde, Departamento de Ações Programáticas Estratégicas. Brasília (DF): Ministério da Saúde; 2018.

27. Brasil. Ministério da Saúde. Secretaria de Atenção Primária à Saúde. Nota Técnica ${ }^{0}$ 10/2020-COCAM/CGCIVI/DAPES/SAPS/MS. Atenção à Saúde do Recém-nascido no contexto da Infecção pelo novo Coronavírus (SARS-CoV-2). Brasília (DF): Ministério da Saúde; 2020

28. Morsch DS, Custódio ZA, Lamy ZC. Psycho-emotional care in a Neonatal Unit during the COVID-19 pandemic. Rev Paul Pediatr. 2020;38:e2020119.

29. Santos LG, Cruz AC, Mekitarian FF, Angelo M. Guia para entrevistas com famílias: estratégia para desenvolver habilidades no enfermeiro novato. Rev Bras Enferm. 2017;70(6):112936 .

30. Global Alliance for Newborn Care (GLANCE). Zero separation. Together for better care! Keep preterm and sick babies close to their parents. Germany:GLANCE; 2020 [cited 2020 Jul 10]. Available from: https://www.glance-network.org/campaign/ 